EPSC Abstracts

\title{
High resolution optical spectroscopy of 2I/Borisov: a surprising similarity to solar system comets
}

Cyrielle Opitom ${ }^{1}$, Emmanuel Jehin ${ }^{2}$, Damien Hutsemékers ${ }^{2}$, Yoshiharu Shinnaka ${ }^{3}$, Jean Manfroid², Philippe Rousselot ${ }^{4}$, Susarla Raghuram5 ${ }^{5}$, Hideyo Kawakita ${ }^{3,6}$, Alan Fitzsimmons ${ }^{7}$, Karen Meech ${ }^{8}$, Marco Micheli ${ }^{9}$, Colin Snodgrass ${ }^{1}$, Bin Yang $^{10}$, and Olivier Hainaut ${ }^{11}$

${ }^{1}$ Institute for Astronomy, University of Edinburgh, Royal Observatory, Edinburgh, UK

${ }^{2}$ Space sciences, Technologies \& Astrophysics Research (STAR) Institute, University of Liège, Liège, Belgium

${ }^{3}$ Koyama Astronomical Observatory, Kyoto Sangyo University, Motoyama, Kamigamo, Kita-ku, Kyoto, Japan

${ }^{4}$ Institut UTINAM UMR 6213, CNRS, Univ. Bourgogne Franche-Comté, Besançon, France

${ }^{5}$ Laboratory for Atmospheric and Space Physics, University of Colorado Boulder, Boulder, CO, USA

${ }^{6}$ Department of Astrophysics and Atmospheric Sciences, Faculty of Science, Kyoto Sangyo University, Motoyama,

Kamigamo,Kitaku, Kyoto, Japan

${ }^{7}$ Astrophysics Research Centre, School of Mathematics and Physics, Queens University Belfast, Belfast, UK

${ }^{8}$ Institute for Astronomy, Honolulu, USA

${ }^{9}$ ESA NEO Coordination Centre, Frascati (RM), Italy

${ }^{10}$ European Southern Observatory, Santiago, Chile

${ }^{11}$ European Southern Observatory, Garching bei München, Germany

2I/Borisov - hereafter 2I - is the first visibly active interstellar comet observed in the solar system. It was discovered on August 20, 2019 and remained visible for several months, permitting observations with various telescopes around the world and in space. We observed 2I with UVES, the high-resolution optical spectrograph mounted on the UT2 telescope at Paranal observatory in Chile, with the objective to characterize its composition. We collected spectra at 12 different epochs between November 15, 2019, and March 16, 2020, covering a heliocentric distance range from 2.1 au pre-perihelion to 2.6 au post-perihelion.

Our observations allowed us to make several key measurements, some being made for the first time in the coma of an interstellar comet. We detected emissions from the radicals $\mathrm{OH}, \mathrm{NH}, \mathrm{CN}, \mathrm{CH}, \mathrm{C}_{2}$, and $\mathrm{NH}_{2}$, the [OI]forbidden oxygen lines at 557.73,630.03, and $636.37 \mathrm{~nm}$, as well as several FeI and NiI lines. We derived the abundance of FeI and NiI in the coma using the model described in [1] and find $\log \mathrm{Q}(\mathrm{NiI})=21.88 \pm 0.07$ molecules $/ \mathrm{s}$ and $\mathrm{Q}(\mathrm{FeI})=21.67 \pm 0.16$ molecules $/ \mathrm{s}$, corresponding to $\log (\mathrm{NiI} / \mathrm{FeI})=0.21 \pm 0.18$. This ratio agrees with the value recently found in solar system comets [1], as shown in Fig. 1.

$\mathrm{NH}_{2}$ ortho and para lines from the ro-vibronic $(0,8,0)$ and $(0,9,0)$ bands were measured and allowed us to derive a $\mathrm{NH}_{2}$ ortho-to-para ratio (OPR) of $3.21 \pm 0.15$, corresponding to an OPR and spin temperature of ammonia of $1.11 \pm 0.08$ and $31^{+10} /-5 \mathrm{~K}$, respectively. These values are consistent with measurements in solar system comets and likely reflect the physico-chemical conditions in the comet inner coma.

We measured the three forbidden oxygen lines and determined the ratio between the intensity of the green [OI] line and the red doublet. We obtained a rather high value $G / R=0.31 \pm 0.05$ close to perihelion, with possibly an increasing trend while the comet moved away from the Sun. This is consistent with the high $\mathrm{CO}$ abundance in the coma of $2 \mathrm{I}$ and the increase of the $\mathrm{CO} / \mathrm{H}_{2} \mathrm{O}$ ratio with the heliocentric distance $[2,3]$. 
Finally, from the $\mathrm{OH}(0-0)$ emission band around $309 \mathrm{~nm}$ we derived a water production rate of $(2.2 \pm 0.2) \times 10^{26}$ molecules/s close to perihelion on December 24 and 26, 2019.

In conclusion, high spectral resolution observations of 2I/Borisov and the associated measurements of the $\mathrm{NH}_{2} \mathrm{OPR}$ and the $\mathrm{Ni} / \mathrm{Fe}$ abundance ratio are remarkably similar to solar system comets. The measured $\mathrm{G} / \mathrm{R}$ ratio is unusually high but consistent with the high abundance ratio of $\mathrm{CO} / \mathrm{H}_{2} \mathrm{O}$ found by other investigators $[2,3]$ and potentially suggesting formation in a colder environment.

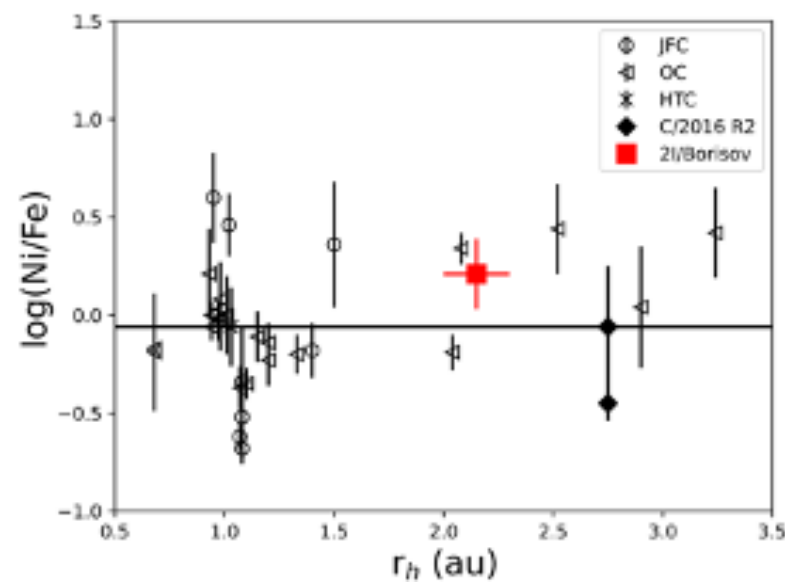

Figure 1: Comparison between the $\log (\mathrm{Ni} / \mathrm{Fe})$ ratio of 2I and the values measured by [1] for a sample of solar system comets (Jupiter Family Comets (JFC), Oort Cloud Comets (OC), and Halley Type Comets (HTC)). The horizontal line represents the average value for solar system comets from [1].

\section{References:}

[1] Manfroid, J., Hutsemékers, D., \& Jehin, E. 2021, Nature, 593, 372

[2] Cordiner, M. A., Milam, S. N., Biver, N., et al. 2020, Nature Astronomy, 4, 861

[3] Bodewits, D., Noonan, J. W., Feldman, P. D., et al. 2020, Nature Astronomy, 4,86 